\section{The impact of obesity on the development and progression of rheumatoid arthritis}

\author{
Axel Finckh, ${ }^{1}$ Carl Turesson ${ }^{2}$
}

Three independent studies examine the impact of obesity on rheumatoid arthritis (RA), either as a risk factor for the development of disease or as a prognostic factor for the severity of disease. ${ }^{1-3}$ Interestingly, these studies have investigated the role of obesity at different stages of the disease, ranging from the 'preclinical phase', to the early RA period, ${ }^{2}$ to the established disease stage. ${ }^{3}$ This is important, as the role played by environmental factors may vary according to disease stage. ${ }^{4}$ An example of the evolving impact of environmental factors during the course of the disease is provided by tobacco smoke, which is the best established risk factor for RA disease development ${ }^{5}$ and associated with the development of severe extraarticular manifestations, ${ }^{6}$ but which may have a protective effect on the progression of joint damage in later stages of the disease. $^{7}$

Obesity is a growing global health problem $^{8}$ and has been associated with increased risk for a number of chronic diseases. There have been conflicting reports on the impact of obesity on the risk of RA, but the majority of studies indicate a positive association in women. ${ }^{9-12}$ As always in the study of lifestyle factors and disease risk, there are methodological issues related to the direction of causality, to recall bias in retrospective investigations and to selection bias. Therefore, prospective population-based studies are needed to formally establish the causal role of obesity in RA.

Lu et $a l^{1}$ report on the effect of overweight or obesity on the development of RA in the Nurses' Health Survey (NHS, enrolment from 1976) and the subsequent NHS II (enrolment from 1989)-two large prospective studies of female registered nurses, which have been used extensively for epidemiological research. Body

\footnotetext{
${ }^{1}$ Division of Rheumatology, University Hospital of Geneva, Geneva, Switzerland; ${ }^{2}$ Rheumatology, Department of Clinical Sciences, Lund University, Malmö, Sweden

Correspondence to Dr A Finckh, Division of Rheumatology, University Hospital of Geneva, Av. Beau-Séjour 26, Geneva 14 CH 1211, Switzerland; axel.finckh@hcuge.ch
}

mass index (BMI) was based on selfreported height and weight, which was assessed every 2 years using mailed questionnaires. Using time-varying BMI, Lu et al observed a trend towards an increased risk of RA in obese nurses (BMI $\geq 30 \mathrm{~kg} / \mathrm{m}^{2}$, according to the WHO definition), with a pooled HR of 1.37 (95\% CI 0.97 to 1.92). The association between obesity and subsequent RA development was stronger in the more recent NHS II. Furthermore, obesity was associated with an increased risk of RA with onset before age 55 . This is in agreement with a previous, population-based control study from Olmsted County, Minnesota, where obesity was associated with an increased risk of RA diagnosis before, but not after, the age of $60 .^{12}$ Finally, in the study by $\mathrm{Lu}$ et al, being overweight (BMI $\geq 25 \mathrm{~kg}$ ) $\mathrm{m}^{2}$, based on retrospective selfassessment) at age 18 was a significant predictor of RA (pooled HR 1.35; 95\% CI 1.10 to 1.66$).{ }^{1}$ Taken together, these results suggest that the relationship between BMI and RA is age dependent, and particularly relevant in younger women.

Given the global rise in the prevalence of obesity, these findings may have major implications for the need for future healthcare utilisation for RA. Indeed, Crowson $e$ a l $^{12}$ estimated that an increase of $20 \%$ in the incidence of RA in women between 1985 and 2007 in Olmsted County, Minnesota, could be attributed to the increase in obesity during this period. $\mathrm{Lu}$ et $a l^{1}$ suggest that secretion of pro-inflammatory cytokines from adipose tissue may contribute to the pathogenesis of RA. However, the mechanisms linking such circulating inflammatory markers to the development of synovitis remain to be elucidated. Alternatively, the association between obesity and RA may reflect residual confounding by other exposures. Although $\mathrm{Lu}$ et al adjusted their analyses for a number of factors, including smoking, alcohol use, hormone-related factors and physical activity, it is still possible that other lifestyle factors related to BMI, such as differences in dietary habits, explain these findings. For example, preliminary results from two prospective studies indicate that a high salt intake ${ }^{13}$ and regular consumption of sugarsweetened soft drinks ${ }^{14}$ may be associated with an increased risk of RA.

Lu et $a l^{1}$ observed similar trends in the association with obesity for seropositive (ie, positive for anti-citrullinated peptide antibodies (ACPA) and/or rheumatoid factor) and seronegative RA, although the impact on ACPA-positive RA was not studied separately. In contrast, several studies have reported an increased risk in obese women for ACPA-negative RA, but not for ACPA-positive RA. ${ }^{10} 11$ This is particularly intriguing, since ACPA-negative $\mathrm{RA}$ is often viewed as a different disease entity, with different underlying pathogenic mechanisms.

Adipocytes in adipose tissue produce bioactive substances, the so-called adipokines. Although their role in the development of RA is not yet fully understood, several adipokines have proinflammatory effects that may play a role in RA disease development. ${ }^{15}$ For example, visfatin activates leukocytes and protects from apoptosis. $^{15}$ Furthermore, blocking visfatin activity has been shown to reduce the severity of arthritis in the collagen-induced arthritis mouse model. ${ }^{16}$ These pathways may be particularly relevant in ACPAnegative RA, where the classic immunological pathways may be relatively less important.

The association between obesity and RA observed in women in the NHS/NHS II and other populations may not apply to men. In the Swedish Epidemiological Investigation of Rheumatoid Arthritis (EIRA), a retrospective case-control study, obesity was associated with a reduced risk of ACPA-positive RA in men. ${ }^{11}$ Furthermore, in a recent prospective study, based on the Malmö Preventive Medicine Project in Sweden, men, but not women, with a high BMI were at a reduced risk of developing RA. ${ }^{17}$ This suggests that hormone-related factors or other sexspecific exposures modify the impact of obesity in RA, and further highlights the potential importance of body fat distribution and diet for RA development.

The role of obesity in RA disease progression and disease severity is less well established. $^{18}$ Obesity in RA has been associated with an increased risk of mortality, cardiovascular comorbidity, total joint replacement, work disability, high medical costs, increased pain and impaired quality of life. ${ }^{19} 20$ Obesity may also negatively influence the long-term evolution of function and disease activity. ${ }^{18} 19$ More recently, it has been suggested that obese patients are less likely to 
respond to anti-tumour necrosis factor $\alpha$ agents (anti-TNFs). ${ }^{21} 22$ Obesity was associated with a decreased likelihood of reaching remission in RA patients treated with anti-TNFs. ${ }^{22}$ In addition, one study has found decreased treatment response to combination therapy with synthetic disease modifying anti-rheumatic drugs (DMARDs) in patients with higher BMIs. $^{23}$

Investigators from Sweden examined whether obese RA patients did in fact have decreased chances of achieving favourable disease outcomes in early disease. ${ }^{2}$ Obese patients were less likely to attain low disease activity or pain remission. ${ }^{2}$ Interestingly, the effect of obesity was notably more pronounced for subjective outcome measures (patient global assessment, tender joint counts) than for objective endpoints (acute phase reactants or swollen joint counts), for which the difference from normal weight patients did not reach statistical significance. Similar observations have been made in other rheumatic diseases. In patients with advanced hip osteoarthritis (OA), increasing BMI was associated with higher pain levels at similar degrees of joint damage. ${ }^{24}$ When adipocytokine concentrations in the synovial fluid of these patients were analysed, the level of joint pain was strongly associated with leptin concentrations, even after correcting for obesity. ${ }^{25}$ Similar observations have been made in hand OA, a condition affecting nonweight-bearing joints. $^{26}$ Overall, these findings suggest that the increased pain observed in women and in obese OA patients may be mediated by adipocytokines. One may hypothesise that specific adipocytokines could also play a role in the arthritic pains in inflammatory arthritides, ${ }^{27}$ and thus account for the association between obesity and pain in RA. ${ }^{2}$

Investigators explored the impact of obesity on structural joint damage in patients with established RA. ${ }^{3}$ Increasing levels of BMI were associated with less radiographic progression over 1 and 2 years after initiation of an anti-TNF. These results are both reassuring and intriguing. Reassuring, because several studies have demonstrated that obese and overweight patients are less likely to respond well clinically to anti-TNF agents. $^{2} 2122$ Therefore, demonstrating that higher residual disease activity in obese patients does not result in irreversible joint damage, is good news. But this finding is also intriguing, as we generally expect a direct relationship between inflammation and structural joint damage.
However, the association between inflammation and structural joint damage appears to be disconnected in patients treated with biologic agents. ${ }^{28}$ Other studies have demonstrated similar findings in RA patients treated with synthetic DMARDs, ${ }^{29}{ }^{30}$ suggesting that the negative association between BMI and radiographic joint damage is not specific to anti-TNF-treated RA. Taken together, these results suggest that a high BMI is associated with an RA phenotype characterised by persistent pain and poor patient reported outcomes, and partially refractory to treatment, whereas low BMI is associated with a phenotype characterised by progressive joint destruction in spite of biologic therapy.

The mechanisms of the association between obesity and low radiographic progression remain elusive, and potentially the causal pathway may be the other way round. Lower BMI could just be a proxy for an intrinsically more aggressive disease, ${ }^{31}$ just as persistent anaemia often reflects chronic inflammation. It should also be recognised that BMI, while readily available and frequently used in epidemiological studies, is a rather crude measure of metabolic status. Changes in the distribution and the properties of adipose tissue over time, alterations in the lean body mass and also ethnic differences, may explain some discrepant results on obesity and RA.

While more research is certainly warranted to understand the precise mechanisms of how obesity affects RA, the clinical implications of these findings are that exercise and weight loss may be important both to prevent metabolic and cardiovascular diseases and also to avoid RA, at least in women. The impact of weight loss programmes in healthy individuals at high risk for the disease, such as first degree family members of RA patients or heavy smokers, should be investigated. Obesity in RA patients seems to be associated with persistent pain. This underlines the importance of lifestyle interventions in the management of patients with RA and other rheumatic diseases.

Contributors Both authors have equally contributed to the analysis and the writing of this editorial.

Provenance and peer review Commissioned; internally peer reviewed.

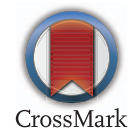

To cite Finckh A, Turesson C. Ann Rheum Dis 2014;73:1911-1913.
Received 14 May 2014

Revised 9 July 2014

Accepted 11 July 2014

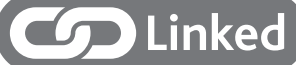

http://dx.doi.org/10.1136/annrheumdis-2014-205544

- http://dx.doi.org/10.1136/annrheumdis-2014-205459

http://dx.doi.org/10.1136/annrheumdis-2013-205094

Ann Rheum Dis 2014;73:1911-1913.

doi:10.1136/annrheumdis-2014-205741

\section{REFERENCES}

1 Lu B, Hiraki L, Sparks JA, et al. Being overweight or obese and risk of developing rheumatoid arthritis among women: a prospective cohort study. Ann Rheum Dis 2014;73:1914-22.

2 Sandberg MEC, Bengtsson C, Källberg H, et al. Overweight decreases the chance of achieving good response and low disease activity in early rheumatoid arthritis. Ann Rheum Dis 2014;73:2029-33.

3 Baker JF, Ostergaard M, George M, et al. Greater body mass independently predicts less radiographic progression on X-ray and MRI over 1-2 years. Ann Rheum Dis 2014;73:1923-8.

4 Gerlag D, Raza K, van Baarsen E, et al. EULAR recommendations for terminology and research in individuals at risk of rheumatoid arthritis (RA): Report from the Study Group for Risk Factors for RA. Ann Rheum Dis 2011:71:638-41.

5 Sugiyama D, Nishimura K, Tamaki K, et al. Impact of smoking as a risk factor for developing rheumatoid arthritis: a meta-analysis of observational studies. Ann Rheum Dis 2010;69:70-81.

6 Turesson C, Schaid DJ, Weyand CM, et al. Association of HLA-C3 and smoking with vasculitis in patients with rheumatoid arthritis. Arthritis Rheum 2006:54:2776-83.

7 Finckh A, Dehler S, Costenbader KH, et al. Cigarette smoking and radiographic progression in rheumatoid arthritis. Ann Rheum Dis 2007;66:1066-71.

8 Finucane MM, Stevens GA, Cowan MJ, et al. National, regional, and global trends in body-mass index since 1980: systematic analysis of health examination surveys and epidemiological studies with 960 country-years and 9.1 million participants. Lancet 2011:377:557-67.

9 Voigt LF, Koepsell TD, Nelson JL, et al. Smoking, obesity, alcohol consumption, and the risk of rheumatoid arthritis. Epidemiology 1994;5:525-32.

10 Pedersen $M$, Jacobsen $S$, Klarlund $M$, et al. Environmental risk factors differ between rheumatoid arthritis with and without auto-antibodies against cyclic citrullinated peptides. Arthritis Res Ther 2006;8:R133.

11 Wesley A, Bengtsson C, Elkan AC, et al. Association between body mass index and anti-citrullinated protein antibody-positive and anti-citrullinated protein antibody-negative rheumatoid arthritis: results from a population-based case-control study. Arthritis Care Res (Hoboken) 2013;65:107-12.

12 Crowson CS, Matteson EL, Davis JM 3rd, et al. Contribution of obesity to the rise in incidence of rheumatoid arthritis. Arthritis Care Res (Hoboken) 2013:65:71-7.

13 Sundström B, Johansson I, Rantapää-Dahlqvist S. Dietary sodium increases the risk for rheumatoid arthritis among smokers-results from a nested case-control study [abstract]. Arthritis Rheum 2013;65(Suppl 10):2271.

14 Hu Y, Costenbader KH, Hu F, et al. Sugar-sweetened soft drink consumption and risk of developing rheumatoid arthritis in women. [abstract]. Arthritis Rheum 2013;65(Suppl 10):112. 
15 Neumann E, Frommer KW, Vasile $\mathrm{M}$, et al. Adipocytokines as driving forces in rheumatoid arthritis and related inflammatory diseases? Arthritis Rheum 2011;63:1159-69.

16 Busso N, Karababa M, Nobile M, et al. Pharmacological inhibition of nicotinamide phosphoribosyltransferase/visfatin enzymatic activity identifies a new inflammatory pathway linked to NAD. PLOS ONE 2008;3:e2267.

17 Turesson C, Bergström U, Pikwer M, et al. High body mass index is associated with a reduced risk of developing rheumatoid arthritis in men [abstract]. Ann Rheum Dis 2013;72(Suppl 3):402.

18 Onuora S. Rheumatoid arthritis. How bad is obesity for RA? Nat Rev Rheumatol. 2012;8:306.

19 Ajeganova S, Andersson ML, Hafstrom I, et al. Association of obesity with worse disease severity in rheumatoid arthritis as well as with comorbidities: a long-term followup from disease onset. Arthritis Care Res (Hoboken) 2013;65:78-87.

20 Wolfe F, Michaud K. Effect of body mass index on mortality and clinical status in rheumatoid arthritis. Arthritis Care Res (Hoboken) 2012;64: 1471-9.

21 Gonzalez-Gay MA, Gonzalez-Juanatey C. Rheumatoid arthritis: obesity impairs efficacy of anti-TNF therapy in patients with RA. Nat rev Rheumatol 2012:8:641-2.

22 Gremese E, Carletto A, Padovan M, et al. Obesity and reduction of the response rate to anti-tumor necrosis factor alpha in rheumatoid arthritis: an approach to a personalized medicine. Arthritis Care Res (Hoboken) 2013;65:94-100.

23 Heimans L, van den Broek M, le Cessie S, et al. Association of high body mass index with decreased treatment response to combination therapy in recent-onset rheumatoid arthritis patients. Arthritis Care Res (Hoboken) 2013;65:1235-42.

24 Lubbeke A, Duc S, Garavaglia G, et al. BMI and severity of clinical and radiographic signs of hip osteoarthritis. Obesity 2009;17:1414-19.

25 Lubbeke A, Finckh A, Puskas GJ, et al. Do synovial leptin levels correlate with pain in end stage arthritis? Int Orthop 2013;37:2071-9.

26 Massengale M, Lu B, Pan JJ, et al. Adipokine hormones and hand osteoarthritis: radiographic severity and pain. PLOS ONE 2012;7: e47860

27 Tian Y, Wang S, Ma Y, et al. Leptin enhances NMDA-induced spinal excitation in rats: a functional link between adipocytokine and neuropathic pain. Pain 2011;152:1263-71.

28 Klarenbeek NB, Guler-Yuksel M, van der Heijde DM, et al. Clinical synovitis in a particular joint is associated with progression of erosions and joint space narrowing in that same joint, but not in patients initially treated with infliximab. Ann Rheum Dis 2010;69:2107-13.

29 Westhoff G, Rau R, Zink A. Radiographic joint damage in early rheumatoid arthritis is highly dependent on body mass index. Arthritis Rheum 2007:56:3575-82.

30 Kaufmann J, Kielstein V, Kilian S, et al. Relation between body mass index and radiological progression in patients with rheumatoid arthritis. J Rheumatol 2003;30:2350-5.

31 Summers GD, Deighton CM, Rennie MJ, et al. Rheumatoid cachexia: a clinical perspective. Rheumatology (Oxford) 2008;47:1124-31. 